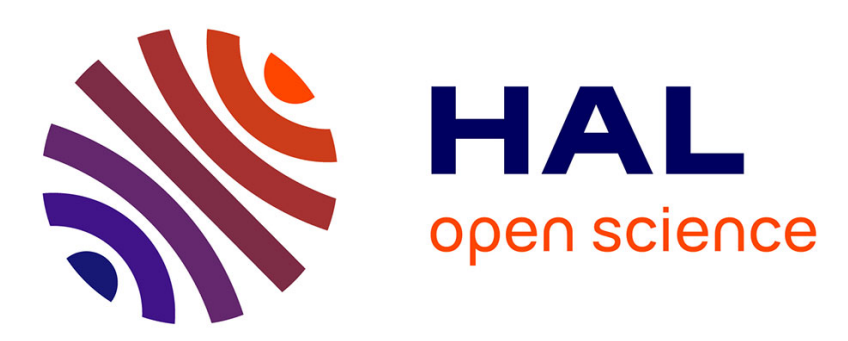

\title{
Nearctis : vers une recherche harmonisée en gestion du trafic et systèmes coopératifs
}

\author{
Juliette Flore Renaud, Pierre-Yves Gilliéron, Winnie Daamen, Ioannis
}

Kaparias, Benjamin Heydecker

\section{To cite this version:}

Juliette Flore Renaud, Pierre-Yves Gilliéron, Winnie Daamen, Ioannis Kaparias, Benjamin Heydecker. Nearctis : vers une recherche harmonisée en gestion du trafic et systèmes coopératifs. RTS - Recherche Transports Sécurité, 2013, 2013 (02), pp.107-123. 10.4074/S0761898013002033 . hal-01670622

\section{HAL Id: hal-01670622 \\ https://hal.science/hal-01670622}

Submitted on 21 Dec 2017

HAL is a multi-disciplinary open access archive for the deposit and dissemination of scientific research documents, whether they are published or not. The documents may come from teaching and research institutions in France or abroad, or from public or private research centers.
L'archive ouverte pluridisciplinaire HAL, est destinée au dépôt et à la diffusion de documents scientifiques de niveau recherche, publiés ou non, émanant des établissements d'enseignement et de recherche français ou étrangers, des laboratoires publics ou privés. 


\title{
Nearctis : vers une recherche harmonisée en gestion du trafic et systèmes coopératifs
}

\section{Nearctis: towards a harmonised research programme on cooperative traffic management}

\author{
Juliette Flore Renaud · Pierre-Yves Gilliéron · Winnie Daamen · Ioannis Kaparias · Benjamin Heydecker
}

Reçu le 17 mai 2012 ; accepté le 23 mai 2013

(C) IFSTTAR et Éditions NecPlus 2013

\begin{abstract}
Résumé Le projet Nearctis est un réseau d'excellence académique constitué d'instituts de recherche européens qui aborde les questions de gestion du trafic et de systèmes coopératifs. Ce réseau a développé un programme de recherche harmonisé qui propose une série de thématiques prospectives dans l'optique d'une croissance de l'intégration des Technologies de l'information et de la communication dans les applications de gestion du trafic.

Cet article décrit en détail deux études de cas : une sur le système de péage urbain à Londres et l'autre sur une gestion intégrée du trafic à une échelle régionale aux Pays-Bas. Ces deux exemples illustrent l'usage des nouvelles technologies et leur potentiel dans des problématiques complexes de gestion du trafic.

L'article conclut sur la perspective de développement d'un institut de recherche en réseau sur ces thématiques prometteuses et surtout sur la nécessité de poursuivre des activités fondamentales en gestion et modélisation du trafic.
\end{abstract}

Juliette Flore Renaud $(\bowtie)$

ERT

c/o Ifsttar, case 24 Avenue François Mitterrand, 69675 Bron, France

e-mail : juliette.renaud@ert-sas.fr

Pierre-Yves Gilliéron $(\triangle)$

Ècole polytechnique fédérale de Lausanne

Laboratoire de Topométrie, Station 18, 1015 Lausanne, Suisse

e-mail : pierre-yves.gillieron@epfl.ch

Winnie Daamen $(\varangle)$

Delft University of Technology, Faculty of Civil Engineering and

Geosciences,

Department of Transport \& Planning

Stevinweg 1, 2628 CN Delft, Pays-Bas

e-mail :W.Daamen@tudelft.nl

Ioannis Kaparias $(\bowtie)$

City University London, Collaborative Transport Hub,

School of Engineering and Mathematical Sciences

Northampton Square, EC1V0HB Londres, Royaume-Uni

e-mail: Ioannis.Kaparias.1@city.ac.uk

Benjamin Heydecker $(\square)$

University College London, Centre for Transport Studies

WC1E 6BT Londres, Royaume-Uni

e-mail : ben@transport.ucl.ac.uk
Mots clés gestion du trafic · systèmes coopératifs . communication inter-véhicules $\cdot$ véhicule intelligent

Abstract Nearctis is an academic network of excellence of European research institutes, which deals with the issues related to the themes of traffic management and cooperative systems. The network has developed a harmonised research programme proposing a series of prospective themes, under the point of view of the ever-increasing integration of Information and Communication Technologies in traffic management applications.

Within this framework, this paper presents two case studies: one on the London Congestion Charging system and the other on integrated traffic management at the regional level in the Netherlands. These two examples illustrate the use of new technologies to deal with complex traffic management problems and demonstrate their potential.

The paper concludes with the development prospects for any research institute in the network on these promising themes, and primarily on the need for continuing the conduct of the fundamental activities in traffic management and modelling.

Keywords Traffic management - Cooperative systems . Vehicle-to-vehicle communications · Intelligent vehicle

\section{Introduction}

La création d'un réseau d'excellence est une aventure académique passionnante et pleine de défis, surtout lorsque l'on s'insère dans une thématique globale comme la gestion $\mathrm{du}$ trafic et les Systèmes de transport intelligents (STI) avec de profondes mutations engendrées par les Nouvelles technologies de l'information et de la communication (NTIC). Cet article présente un volet des activités du réseau Nearctis, créé en 2008 , avec l'établissement d'un programme harmonisé de recherche qui s'appuie sur une démarche scientifique menée conjointement avec de multiples partenaires provenant des institutions, de 
l'industrie, des gestionnaires de routes et du monde de la recherche. Afin d'illustrer la thématique de la gestion du trafic et des systèmes coopératifs, l'article présente en détail deux études de cas dont les enjeux sont représentatifs des futurs défis de recherche. Finalement, on présente les perspectives d'évolution du réseau Nearctis qui sera transformé en un institut virtuel de recherche, dont une des missions principales sera la promotion de son programme de recherches, à côté des activités de formation et de gestion de précieuses ressources spécifiques à la recherche en gestion du trafic.

\section{Le projet Nearctis}

Nearctis (A Network of Excellence for Advanced Road cooperative traffic management in the Information Society) est un projet financé par la Commission Européenne au titre du $7^{e}$ Programme-cadre de recherche et développement. L'objectif de ce projet est de créer une dynamique de collaboration entre acteurs de l'excellence en gestion du trafic, qui amènera le réseau à se pérenniser. Doté d'un budget total de 3,134 millions d'euros (dont 2,5 millions de financement $\mathrm{CE}$ ), il était au départ prévu pour durer 4 ans, mais a bénéficié d'une extension de durée d'un an et s'achèvera le 30 juin 2013. Huit grands instituts européens sont partenaires du projet : il s'agit de l'Ifsttar (Institut Français des sciences et technologies des transports, de l'aménagement et des réseaux), du DLR (Deutschen Zentrums für Luft-und Raumfahrt), l'EPFL (École Polytechnique Fédérale de Lausanne), d'ICL (Imperial College London), de TUC (Technical University of Crete), de TU Delft (Delft University of Technology), de University of Southampton et d'UCL (University College London). La coordination du projet est assurée par ERT, une filiale de l'Ifsttar qui est chargée du montage et du management des projets européens.

Les activités de Nearctis sont centrées autour des problématiques de gestion du trafic et d'optimisation, avec une attention particulière portée sur les systèmes coopératifs pour une meilleure régulation du trafic routier. Cependant, le projet va au-delà, et se propose de développer des concepts capables de faire face aux défis liés à la sécurité, consommation énergétique, impacts environnementaux, congestion ; bref tous les obstacles à la mobilité.

Nearctis, comme tous les réseaux d'excellence européens, a pour vocation, par le biais de ses différentes activités, de favoriser la création, à l'issue du projet, d'un « institut virtuel » qui sera un point de référence en Europe sur les thématiques de gestion du trafic. Cet «institut virtuel », ou institut en réseau, qui prendra très certainement la forme d'une association de droit français, devra relever de nombreux défis, entre autres :
- identifier des activités et des thèmes de recherche innovants pour attirer des membres, alors même que les financements européens auront pris fin ;

- réaliser un business plan qui permette d'assurer la pérennisation de la future structure ;

- créer une synergie forte entre professionnels européens.

Neufs partenaires académiques européens sont officiellement impliqués dans le projet ; afin de lui assurer une pluralité de compétences et des contributions suffisamment éclectiques pour répondre à son objectif d'excellence, Nearctis s'est de plus doté d'un réseau de partenaires associés. Venus du monde de la recherche et de l'industrie, ils sont localisés partout dans le monde. Leur participation aux activités du projet se fait sur la base du volontariat.

Afin de réaliser ses objectifs, Nearctis a mis en place différents groupes de travail (Work Packages). Ils produisent des livrables qui sont accessibles à tous, mais ils sont également actifs dans l'organisation d'événements de dissémination des connaissances et dans des actions éducatives destinées aux jeunes chercheurs afin de créer une dynamique entre les instituts impliqués dans le projet.

\section{Work Package 1 - programme de recherche : définition et coordination}

L'objectif de ce groupe de travail est d'établir un état des lieux des avancées scientifiques du domaine, puis de proposer, grâce aux éléments récoltés auprès des différents partenaires et partenaires associés, un programme de recherche harmonisé qui constitue une feuille de route pour les instituts européens concernés par la gestion du trafic, ainsi que pour les décideurs de l'Union Européenne [4].

Les trois livrables produits pendant le projet sont disponibles sur son site internet à l'adresse suivante : http://www.nearctis.org/home/resources-desk/deliverables/

\section{Work Package 2 - étude de cas : spécifications et évaluations}

L'objectif de ce groupe de travail est d'identifier des études de cas pouvant être potentiellement conduites. Le work package tente aussi de développer des spécifications et pré-évaluations pour ces études de cas [5].

Les trois livrables produits sont disponibles sur son site internet à l'adresse indiquée ci-dessus.

\section{Work Package 3 - éducation, formation et dissémination}

Ce groupe de travail est central pour encourager une dynamique de mise en réseau de l'excellence et pour 
assurer la circulation des connaissances. Outre les trois livrables produits sur ce sujet (disponible à l'adresse mentionnée ci-dessus) qui résument les activités du groupe de travail, les partenaires ont été impliqués dans l'organisation de trois écoles d'été, et de workshops annuels. Nearctis s'est également doté d'un programme de mobilité des chercheurs finançant des séjours de courte durée dans des établissements partenaires, ainsi que d'un programme de 5 thèses incluant là aussi une mobilité des étudiants.

\section{Work Package 4 - capitalisation, ressources partagées}

Rassembler les ressources dont se sont dotés au fil du temps les différents partenaires constitue un élément important du réseau d'excellence ; en effet, cela permet de renforcer les capacités de recherche en Europe. La mission de ce groupe de travail a été d'inventorier ces ressources, de mettre en œuvre un cadre commun pour leur utilisation, ainsi qu'un support informatique rassemblant différentes bases de données. Enfin, des synergies ont été identifiées afin d'utiliser au mieux ces ressources. Les bases de données identifiées sont disponibles pour nos partenaires et partenaires associés sur le site Web du projet (www.nearctis.org).

\section{Work Package 5 - coordination et management du réseau}

Ce groupe de travail s'assure de la bonne marche du projet, ainsi que du respect des obligations contractuelles vis-à-vis de notre financeur. Il est également en charge de dessiner les grandes lignes du futur réseau virtuel qui succédera à Nearctis, dont les résultats sont présentés lors de sa conférence finale en juin 2013.

\section{Études de cas : identification et évaluation}

En complément du programme harmonisé de recherche, une autre activité de Nearctis est celle de la pré-évaluation d'études de cas pertinentes offrant un potentiel de développement. Les études de cas sélectionnées doivent remplir les conditions suivantes :

- représenter des problèmes complexes qui peuvent bénéficier de l'application des techniques novatrices issues de la recherche (par exemple, celles identifiées dans le cadre du programme de recherche harmonisé) ;

- être représentatives des problèmes et des stratégies de gestion du trafic apparaissant dans plusieurs réseaux routiers en Europe ;

- comprendre des nouveaux défis de recherche, en particulier dans le contexte des NTIC.
Ainsi, ces études de cas ont été déployées dans des régions où l'on avait identifié la nécessité de recourir aux systèmes et méthodes de gestion du trafic, en considérant différentes échelles géographiques ainsi que des conditions particulières d'état du trafic.

Les domaines d'études sélectionnés sont décrits de manière uniforme : une première partie résume le système en question et la méthode appliquée, une seconde partie procède à une analyse des forces et faiblesses, et la dernière partie explore les opportunités d'amélioration amenées par les STI coopératifs actuels et émergents. En tout, cinq thèmes ont été étudiés :

Services globaux : ils sont conçus pour la gestion avancée du trafic dans des grandes zones, au niveau des utilisateurs individuels. Ces études de cas abordent, par exemple, les systèmes de péage implicites (shadow toll systems), les méthodes de gestion de flottes de véhicules, les techniques de guidage porte-à-porte et les pratiques de coordination au niveau du réseau.

Grands couloirs autoroutiers : ils se réfèrent aux trajets de longue distance. Ces études de cas abordent quelques mises en pratique représentatives des méthodes de gestion du trafic sur autoroutes. Ce sont, par exemple, les systèmes de guidage et d'information au conducteur, les méthodes de gestion des accès, les techniques de limitation de vitesse variable et la gestion du trafic dans les tunnels.

Réseaux urbains denses : ils sont définis à l'échelle d'une ville entière, incluant diverses catégories de routes et de modes de transport. Ces études de cas couvrent les concepts d'opération des centres de gestion du trafic, les méthodes de gestion des feux, les pratiques de péage urbain et les méthodes appliquées pour donner, par exemple, la priorité aux véhicules des transports en commun.

Réseaux locaux d'artères principales : ces études de cas contiennent des exemples de gestion des voies afin de réduire les embouteillages récurrents, et des pratiques de gestion d'incidents pour diminuer les embouteillages non-récurrents.

Réseaux partagés multimodaux et multi-usagers : c'est la stratégie d'utilisation d'une même voie publique de circulation par des différents modes et usagers. Ces études de cas couvrent les méthodes de gestion de la priorité des bus aux carrefours basées sur la localisation automatique de véhicules, les pratiques intégrées de priorité des bus, les systèmes de signalisation électronique pour les piétons et la gestion du trafic multimodal à l'échelle d'un réseau.

Le compte rendu complet des études de cas décrites dans ce projet est disponible dans les rapports détaillés de Nearctis [2]-[3]. Cet article se concentre sur deux exemples choisis : la gestion intégrée du trafic à l'échelle d'une agglomération (Amsterdam), dans le cadre des services globaux ; et sur les systèmes de péage urbain, dans 
le cadre des réseaux urbains denses, avec l'exemple de Londres. Le choix de ces deux études de cas est basé sur le fait qu'elles forment des exemples représentatifs des applications de STI dans des environnements périurbains et urbains respectivement. Les deux études démontrent les avantages apportés par l'identification des domaines et perspectives d'une recherche harmonisée et du potentiel des systèmes coopératifs dans la gestion des systèmes de transport dans des conditions cadres qui peuvent varier d'un pays à l'autre ou entre différentes villes.

\section{Étude de cas 1 : gestion intégrée du trafic à l'échelle d'une agglomération}

\section{Présentation}

Les objectifs pour le trafic et le transport aux Pays-Bas d'ici à 2020 sont décrits dans le Nota Mobiliteit (la note sur la mobilité). Pour faire face aux problèmes de circulation actuels et futurs ce message sur la mobilité présente des objectifs ambitieux : «de la mobilité porte à porte plus rapide, plus propre et plus sûre ». Trois piliers interdépendants sont identifiés pour aider à la réalisation de ces objectifs : la construction de l'infrastructure routière, la tarification de l'usage de l'infrastructure et l'utilisation de l'infrastructure.

Les piliers « construction » et « tarification » sont des instruments puissants pour résoudre les problèmes de congestion, mais ils ont leurs limites à court terme. D'une part, en raison de la réglementation sur la qualité de l'air et d'autre part d'un ajournement de l'entretien, la construction et l'élargissement des routes sont retardés. Parce que la mise en œuvre de la tarification routière a été retardée pour des raisons techniques et que la congestion augmente toujours, le ministère néerlandais a formulé une politique claire sur la transition vers une utilisation optimale du réseau routier néerlandais par la gestion du trafic routier dans le pilier « utilisation» [6]. Cette politique est soutenue par la volonté d'investir structurellement dans la gestion du trafic routier - étant donné que le rapport coût-efficacité des mesures en général ne peut pas être démontré, en particulier lorsqu'il s'agit de coordination et d'intégration des mesures. C'est l'une des principales motivations qui a permis d'initier l'étude pilote dans la région d'Amsterdam.

Avant de définir les mesures, leur coordination et leur intégration dans la région d'Amsterdam, un aperçu a été fait des goulots d'étranglements dans le réseau routier. L'accent a été mis sur les goulots d'étranglement qui sont présents

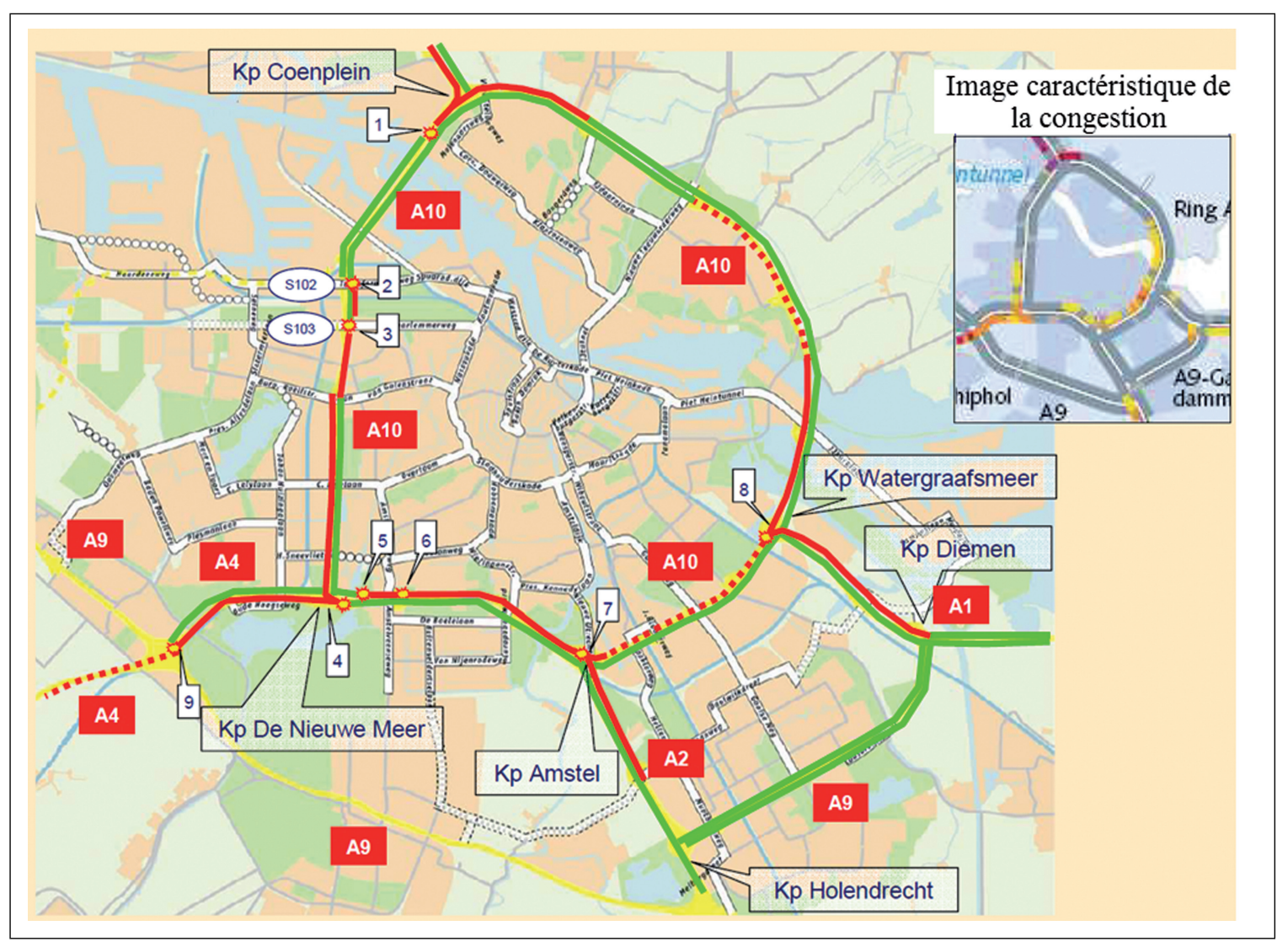

Figure 1 Réseau routier et lieux initiaux de la congestion dans la région Amsterdam 
quotidiennement, avec un impact direct ou indirect sur le trafic sur les autoroutes A9, A10 et N201 et conduisant à de graves retards sur les connexions ou les itinéraires alternatifs. Les causes sont les points de départ de problèmes que l'on retrouve dans le flux de trafic, par exemple lorsque le trafic s'insère ou en présence d'un étranglement de la route. La conséquence est généralement une formation de congestion. La figure 1 montre les lieux initiaux de la congestion dans la région d'Amsterdam pendant l'heure de pointe du matin [7]. Une illustration similaire a été établie pour l'heure de pointe de l'après-midi.

Le réseau n'est pas affecté par une unique source de congestion, il en existe de multiples, à la fois sur le réseau autoroutier et sur le réseau urbain. Par conséquent, le réseau routier de la région d'Amsterdam est très approprié pour effectuer une étude pilote pour l'évaluation de la gestion intégrée de trafic à l'échelle d'une agglomération.

L'objectif concret de l'étude pilote consiste à déterminer les effets de la gestion intégrée du trafic à l'échelle d'une agglomération. Dans cette étude, la gestion de trafic est destinée à réduire la croissance de la congestion, et donc la perte de temps des véhicules sur le réseau dans toute la région, pour finalement améliorer la prévisibilité des temps de parcours. Si l'étude pilote est réussie, il est prévu d'appliquer la gestion intégrée du trafic à l'échelle d'une agglomération à d'autres régions urbaines, en tenant compte des conditions spécifiques de chaque région.

Les principaux objectifs de l'étude pilote sont :

1. comprendre le fonctionnement et l'utilité d'un certain nombre de nouveaux outils pour la gestion de trafic ;

2. démontrer la faisabilité et l'efficacité de la gestion intégrée de trafic à l'échelle d'une agglomération. Poser les bases pour ce type de gestion pour les autorités routières et pour les usagers de la route. Estimer le potentiel de la gestion intégrée de trafic à l'échelle d'une agglomération pour l'utiliser ailleurs dans le pays.

Les hypothèses de l'élaboration de l'étude pilote sont les suivantes :

- montrer de l'ambition, sans prendre de risques excessifs. Dans la mesure du possible, s'appuyer sur les expériences nationales et internationales traitant des aspects partiels de la gestion intégrée de trafic à l'échelle d'une agglomération ;

- s'appuyer sur les pratiques (inter)nationales et, en cas de mise en œuvre nationale, recourir à l'expertise et à l'expérience internationale ;

- se référer aux objectifs et aux contraintes régionales pour élaborer la gestion intégrée de trafic à l'échelle d'une agglomération pour la situation régionale à Amsterdam ;

- limiter les réalisations aux ajustements qui sont possibles et techniquement réalisables en impliquant les parties concernées afin de prouver explicitement le concept ;

- limiter les réalisations aux ajustements lorsque des tests et des évaluations sont possibles ;

- prévoir une communication claire de l'étude pilote pour les usagers de la route et les autres parties intéressées.

\section{Les principes de la gestion intégrée de trafic à l'échelle d'une agglomération}

Depuis longtemps, des mesures de gestion du trafic sont prévues afin d'augmenter l'utilisation de l'infrastructure - comme les feux, la gestion des accès et les panneaux à messages variables. Jusqu'à présent, ces mesures sont utilisées pour résoudre des problèmes au niveau local sur le réseau routier, en particulier sur les routes principales. L'innovation de la gestion intégrée du trafic à l'échelle d'une agglomération intègre diverses mesures qui sont prises et contrôlées sur un réseau régional, non seulement sur le réseau routier principal, mais aussi sur le réseau routier sous-jacent, et de manière cohérente. De cette façon, les effets de la gestion ont un impact plus important : ils permettent d'éviter que les problèmes se déplacent, et, si nécessaire, d'utiliser de façon optimale la capacité disponible de l'ensemble du réseau.

La figure 2 donne un aperçu du système développé pour la gestion intégrée de trafic à l'échelle d'une agglomération, y compris les différentes unités logiques, les différents niveaux considérés, etc. Les principaux éléments du système sont expliqués brièvement ci-dessous.

Le cadre logique de gestion se compose des trois types d'éléments clés :

1. des unités de contrôle logiques (pour mesurer l'état du trafic, sans actions) ;

2. des unités de supervision;

3. des unités de gestion logiques (pour agir sur l'état du trafic).

Chacun de ces types d'éléments effectue des tâches spécifiques. Il est important de comprendre que toutes ces unités logiques sont définies pour des niveaux de regroupement multiples (Figure 2). Le terme « regroupement » est utilisé pour décrire les différents niveaux de coordination nécessaires pour résoudre les problèmes croissants et selon leur gravité. Afin de les rendre opérationnels, on définit les niveaux de regroupement suivants du système de la gestion intégrée de trafic à l'échelle d'une agglomération :

1. points ou des mesures locales ;

2. artères (mesures coordonnées); 


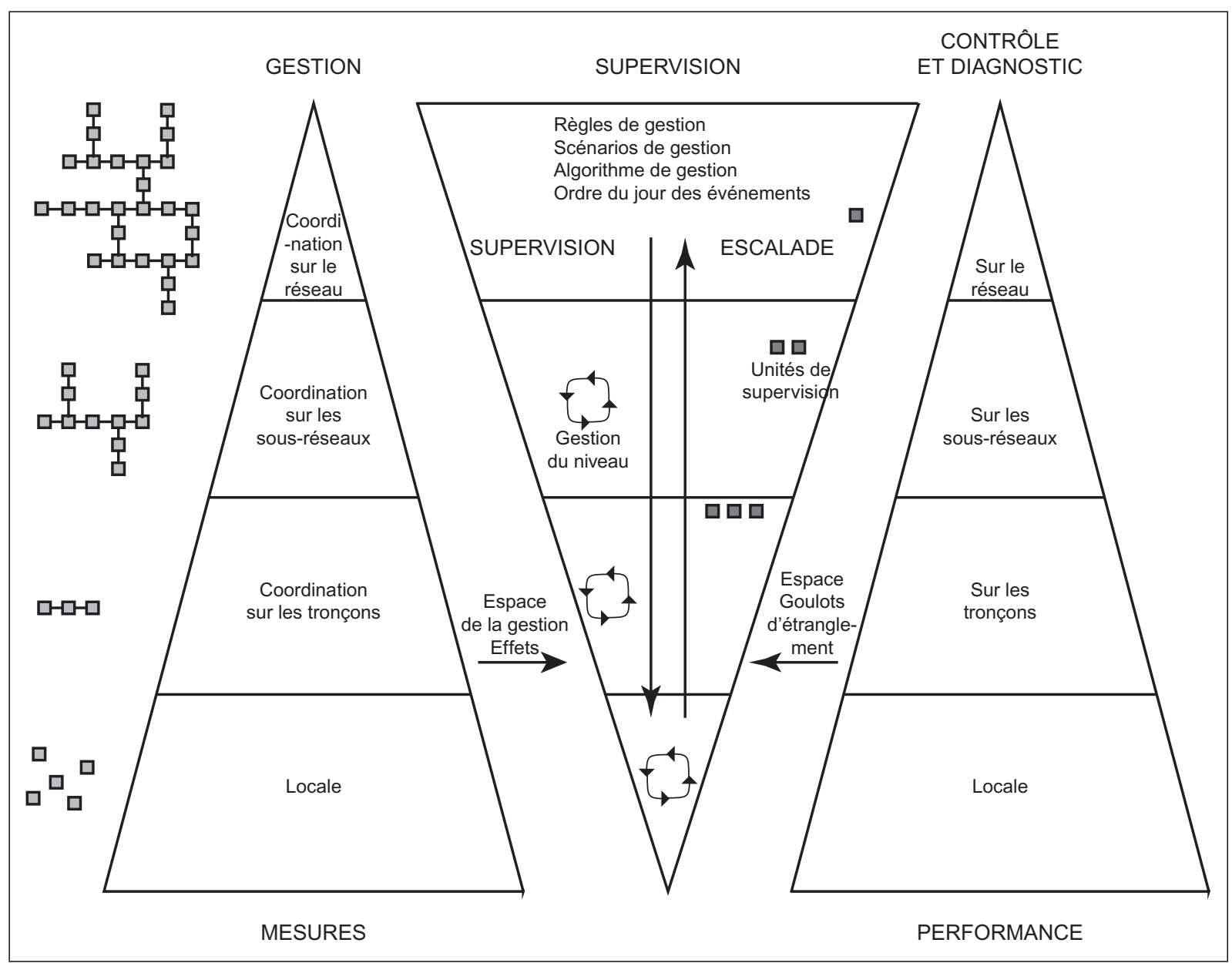

Figure 2 Cadre pour la gestion intégrée de trafic à l'échelle d'une agglomération caractérisé par les éléments « gestion », « supervision » et « contrôle et diagnostic »[8]

3. sous-réseaux (clusters de grandes artères) ;

4. réseau.

Ainsi, le système n'incorpore pas seulement la gestion intégrée du trafic et des mesures de coordination le long d'une artère, mais également à un niveau supérieur. Des unités peuvent être définies afin de prendre des mesures appropriées pour résoudre ou prévenir les problèmes en recourant à une plus grande partie du réseau. Dans le même temps, la hiérarchie maintient la complexité du système à un niveau modéré.

Les unités logiques de gestion influencent les flux de trafic. Elles sont directement liées aux mesures physiques de la gestion de trafic (gestion des accès, feux, etc.). Les unités logiques de gestion au niveau du regroupement le plus bas (points) fonctionnent de manière autonome lorsque des problèmes se produisent au niveau local dans le réseau. Lorsque les problèmes deviennent plus graves, les unités logiques de gestion de niveau supérieur prennent le relais, en principe après l'intervention d'un superviseur, et le niveau de coordination augmente. Les unités de gestion disposent du niveau approprié de connaissances pour communiquer avec l'unité de supervision qui maintient la performance celles-ci, afin de donner des instructions et de déterminer quand il est nécessaire de s'étendre sur un plus haut niveau de contrôle. À partir du niveau des artères, les unités de gestion fournissent en permanence à l'unité de supervision leur espace (prévu) de contrôle et les effets (prévus) qu'elles peuvent réaliser.

L'unité de supervision vise à trouver une configuration optimale de coordination pour résoudre un problème imminent en combinant les informations concernant les effets proposés par les unités de gestion logique et l'effet (exigé) identifié par les unités logiques de contrôle (la demande).

Les unités logiques de contrôle surveillent les opérations de trafic sur le réseau routier au niveau du regroupement (point, tronçon, sous-réseau, réseau) qui leur est attribué. 


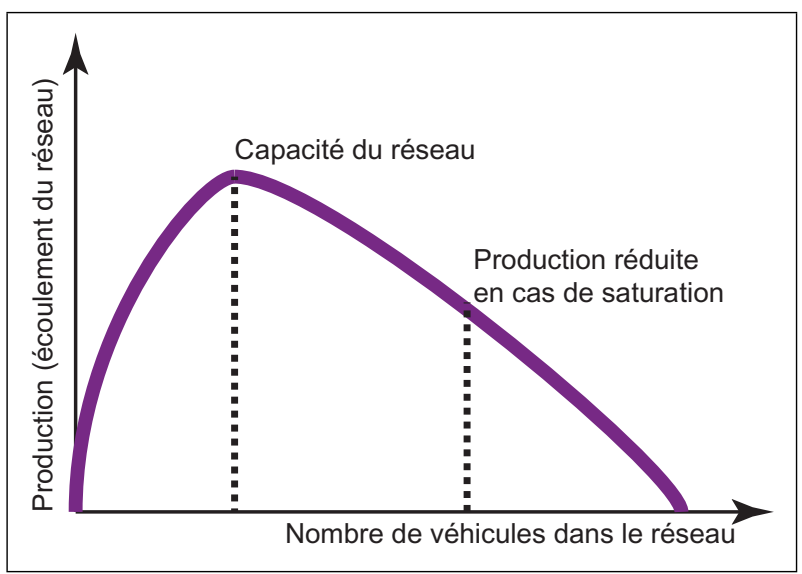

Figure 3 Le diagramme fondamental macroscopique

Elles fournissent constamment aux unités de gestion et à l'unité de supervision des informations concernant les goulots d'étranglement et les capacités de stockage dans des parties spécifiques du réseau. À cet effet, elles collectent les données de flux de trafic au moyen de détecteurs et elles manipulent ces données, si nécessaire.

Pour déterminer les goulots d'étranglement et l'espace de contrôle au niveau des artères et des sous-réseaux, on utilise un diagramme fondamental macroscopique [9]-[10]. Cela donne la relation entre le nombre de véhicules dans le réseau et l'écoulement dans le réseau, présenté d'une manière schématique (Figure 3). Si le nombre de véhicules présents dans le réseau augmente au-delà d'une valeur critique, alors le nombre de véhicules qui atteignent leur destination ou qui quittent le réseau sur une période de temps donnée diminue à cause de blocages internes. Les deux rôles du diagramme fondamental macroscopique dans la gestion intégrée de trafic à l'échelle d'une agglomération sont : d'une part le diagnostic (est-ce qu'il y a un goulot d'étranglement au niveau du réseau ?) et d'autre part la résolution de problème (est-ce qu'il y a un espace de contrôle dans la partie concernée du réseau?).

\section{L'élaboration pour la région d'Amsterdam}

Le point de départ pour la gestion intégrée du trafic à l'échelle d'une agglomération dans la région d'Amsterdam est donné dans la «Vision du réseau Nord Hollande » [11]. Dans ce message, les routes qui font partie du réseau disponible pour la gestion du trafic sont stipulées dans les grandes lignes.

Des routes désignées peuvent être gérées lors des situations habituelles ou exceptionnelles : c'est le réseau routier disponible pour la gestion du trafic. Ensuite, une stratégie de gestion a fondée sur les principes politiques de la Vision du réseau est développée. Ceci détermine la capacité routière de réserve qui est distribuée pendant une surcharge du réseau régional. Pour la stratégie de gestion, on a prédéterminé des priorités fixées dans une « carte de priorité » (Figure 4). Cette carte indique la qualité souhaitée pour les tronçons de route par rapport aux tronçons de route du voisinage. En outre, la stratégie tient compte des fonctions spécifiques de l'intégralité des différentes routes dans le réseau prévues dans une «carte de fonctions ». Tant

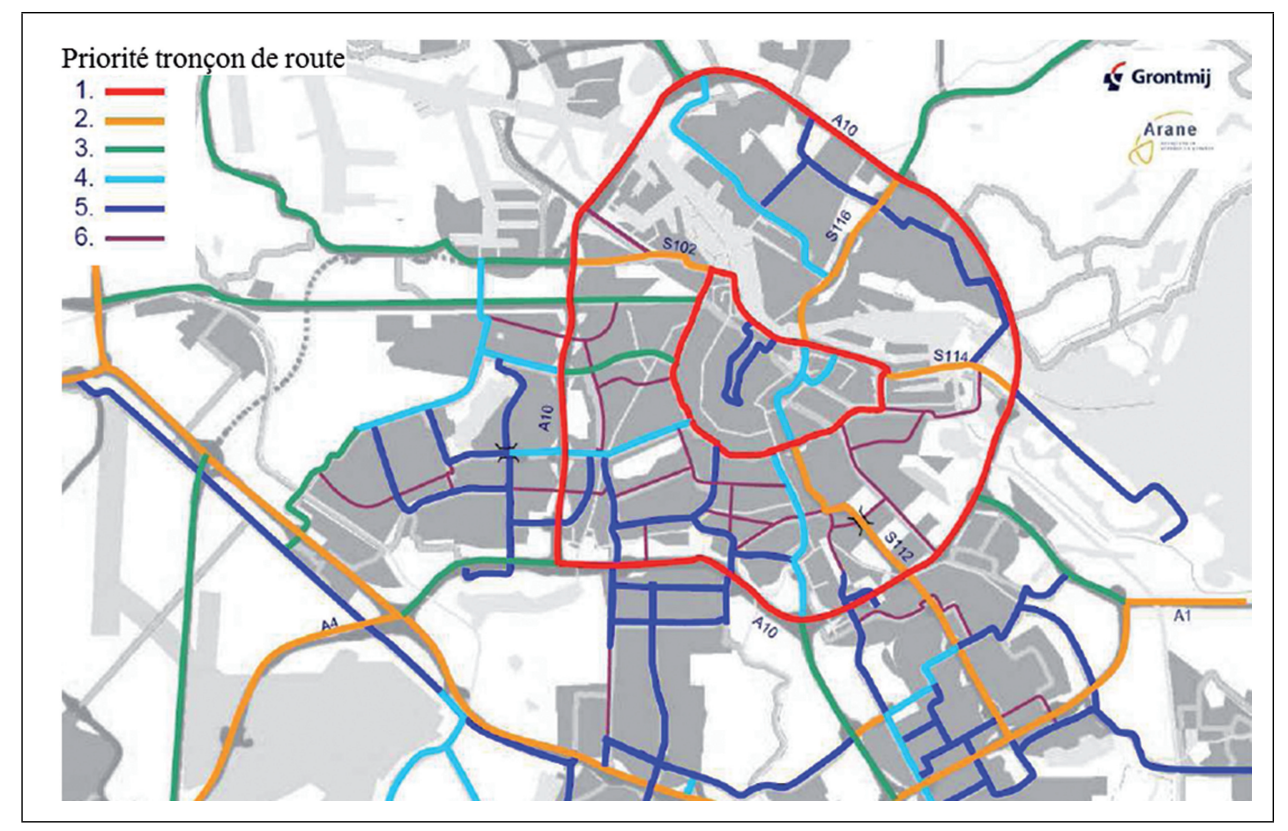

Figure 4 Priorités des routes sur le réseau routier disponible dans la région d'Amsterdam [8] 


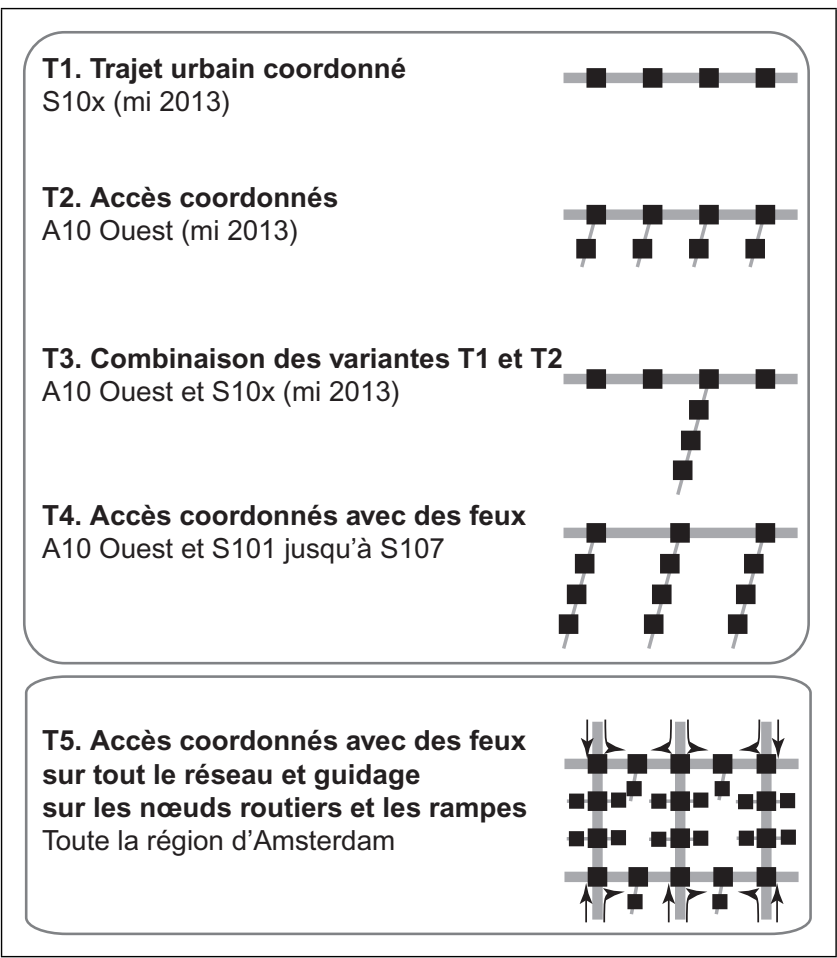

Figure 5 Représentation schématique des mesures dans le réseau d'Amsterdam

la carte de priorités que la carte de fonctions sont alignées avec les souhaits des partenaires régionaux.

Sur cette base, les principes de gestion sont formulés, en indiquant comment la gestion intégrée du trafic à l'échelle d'une agglomération facilite ou améliore les opérations de trafic dans le réseau. Un «paquet de mesures » a été mis au point afin de permettre une gestion efficace. Les grandes lignes pour la mise en œuvre de ces mesures sont décrites dans la «philosophie de gestion». Toutes les composantes de cette structure sont importantes pour gérer les situations habituelles et exceptionnelles.

Pour la région d'Amsterdam, les mesures de crise suivantes sont testées dans l'étude pilote (Figure 5):

T1 : coordination de la gestion du trafic sur une artère urbaine.

$\mathrm{T} 2$ : coordination des gestions des accès sur A10 Sud et A10 Ouest.

T3/T4 : mise en œuvre coordonnée du T2 et (T3: un, T4 : multiples) $\mathrm{T} 1$.

T5 : coordination complète du (sous-) réseau.

Pour T1 (artères urbaines), l'idée est que le niveau de coordination dépend de la charge du réseau. Pour les petites charges, aucune coordination n'est nécessaire et la gestion locale est probablement optimale. Pour les charges légèrement plus élevées, le phénomène de déplacement en peloton (platooning) nécessite des formes spécifiques de coordination (p. ex. les ondes vertes). Lorsque les charges deviennent encore plus élevées, des algorithmes de gestion du réseau en temps réel sont exploités pour optimiser les conditions d'écoulement.

Pour T2, la gestion locale des accès est déployée aussi longtemps que nécessaire. Une fois qu'une demande de coordination se pose, les algorithmes de type HERO [12]-[16] (utilisé, par exemple, sur l'autoroute Monash dans la région de Melbourne) sont mis en ouvre afin d'utiliser l'espace de stockage en amont de la gestion des accès pour permettre le dosage prolongé. Contrairement aux versions antérieures de gestion coordonnée des rampes, la rampe et les carrefours en amont sont utilisés pour stocker le trafic qui n'est pas encore autorisé à entrer dans la route principale. L'idée clé est que le rapport entre la longueur de la queue (sur l'accès et sur les carrefours en amont) et la longueur maximale de la queue est maintenu égal pour tous les accès concernés par la coordination.

Pour T3, une artère urbaine sous gestion (T1) est reliée à l'autoroute sous gestion (T2). L'idée générale est que le régime de la coordination $\mathrm{T} 2$ détermine l'entrée sur la route principale (par exemple en utilisant HERO), tandis que l'artère urbaine sous gestion (T1) tente de maintenir les conditions sur l'artère aussi efficace que possible. Plutôt que d'utiliser l'espace maximum de stockage sur l'accès, on considére le nombre de véhicules qui peuvent être stockés sur l'artère sans en réduire sévèrement la performance. T4 est essentiellement le même, mais dans ce cas, plusieurs lignes $\mathrm{T} 1$ sont reliées à un $\mathrm{T} 2$.

La spécification précise des algorithmes de gestion (pour les T1 et T2) est achevée mi-2012. En plus, les actionneurs doivent être modifiés (techniquement) pour permettre la communication appropriée entre le contrôleur et le centre de gestion du trafic.

\section{Potentiel pour les systèmes coopératifs}

Au début, l'étude pilote ne permettait qu'une manière de gérer le trafic, c'est-à-dire en utilisant des systèmes de contrôle et d'information placés au bord de la route. Récemment, on a ajouté une nouvelle possibilité en prévoyant d'utiliser des systèmes coopératifs. L'idée est de tester les deux types de mesures jusqu'à un certain degré et d'une manière intégrée.

\section{Étude de cas 2 : système de péage urbain avec l'exemple de Londres}

Sur le site internet de Transport for London (TfL), il est noté que quand Ken Livingstone est entré en fonction 
comme maire de Londres en l'an 2000 : (i) Londres était affligé par la pire circulation du trafic dans le Royaume-Uni et une des pires en Europe ; (ii) les automobilistes au centre de Londres passaient $50 \%$ de leur temps dans des embouteillages, (iii) un volume de trafic équivalent à 25 voies autoroutières entrait au centre de la ville tous les jours de la semaine ; et (iv) il était estimé que Londres perdait entre 2 et 4 millions de livres sterling (£) (entre 3 et 5 millions d'euros) par semaine à cause du temps perdu dans les embouteillages. C'est dans ce contexte-là que le système de péage urbain London Congestion Charging (LCC) a été introduit à Londres le 17 février 2003.

\section{Présentation}

Le LCC est classifié comme un système de péage zonal, dans lequel les véhicules localisés à l'intérieur d'une zone doivent payer un montant précis. La zone définie le 17 février 2003 était de $22 \mathrm{~km}^{2}$, englobe les centres principaux de commerce, d'administration et de divertissement - c'est-à-dire l'intégralité de la City of London (zone financière) et du West End (le plus important centre de divertissement et de commerce) ainsi que 136 000 habitants. La zone a été élargie à l'ouest (Western
Extension Zone - WEZ) le 19 février 2007 sur une surface totale de $37 \mathrm{~km}^{2}$, mais a ensuite été réduite à ses limites d'origine le 24 décembre 2010, suite à une enquête publique (Figure 6).

Le projet initial prévoyait le paiement de $£ 5(7,5 €)$ par jour et par propriétaire/utilisateur de véhicule situé sur la voie publique dans la zone, et ce aux heures pendant lesquelles le système est opérationnel (de $7 \mathrm{~h}$ a $18 \mathrm{~h} 30$, $\mathrm{du}$ lundi au vendredi). Les frais se sont élevés à $£ 8$ $(11 €)$ en juillet 2005 puis à $£ 10$ (12 €) en janvier 2011, et l'introduction de la WEZ le 19 février 2007 (ensuite supprimée) a aussi modifié les heures d'opération (de $7 \mathrm{~h}$ à $18 \mathrm{~h}$ en semaine). À noter que les frais ne s'appliquent pas les samedi et dimanche, les jours fériés, et entre $18 \mathrm{~h}$ et $7 \mathrm{~h}$, ainsi qu'aux jours désignés libres (comprenant la période entre le jour de Noël et le jour de l'An). Le LCC peut aussi être suspendu par TfL en raison d'incidents sérieux (par exemple, la suspension du 7 et 8 juillet 2005 en réponse aux attentats terroristes sur le réseau des transports en commun, et la suspension du 2 février 2009, en raison d'enneigement exceptionnel).

Des frais de $£ 10$ sont facturés jusqu'à minuit le jour du trajet dans la zone, soit d'avance ou après. Les frais peuvent aussi être payés le jour suivant à un coût majoré de $£ 12$ $(15 €)$. Le paiement peut être effectué de plusieurs manières

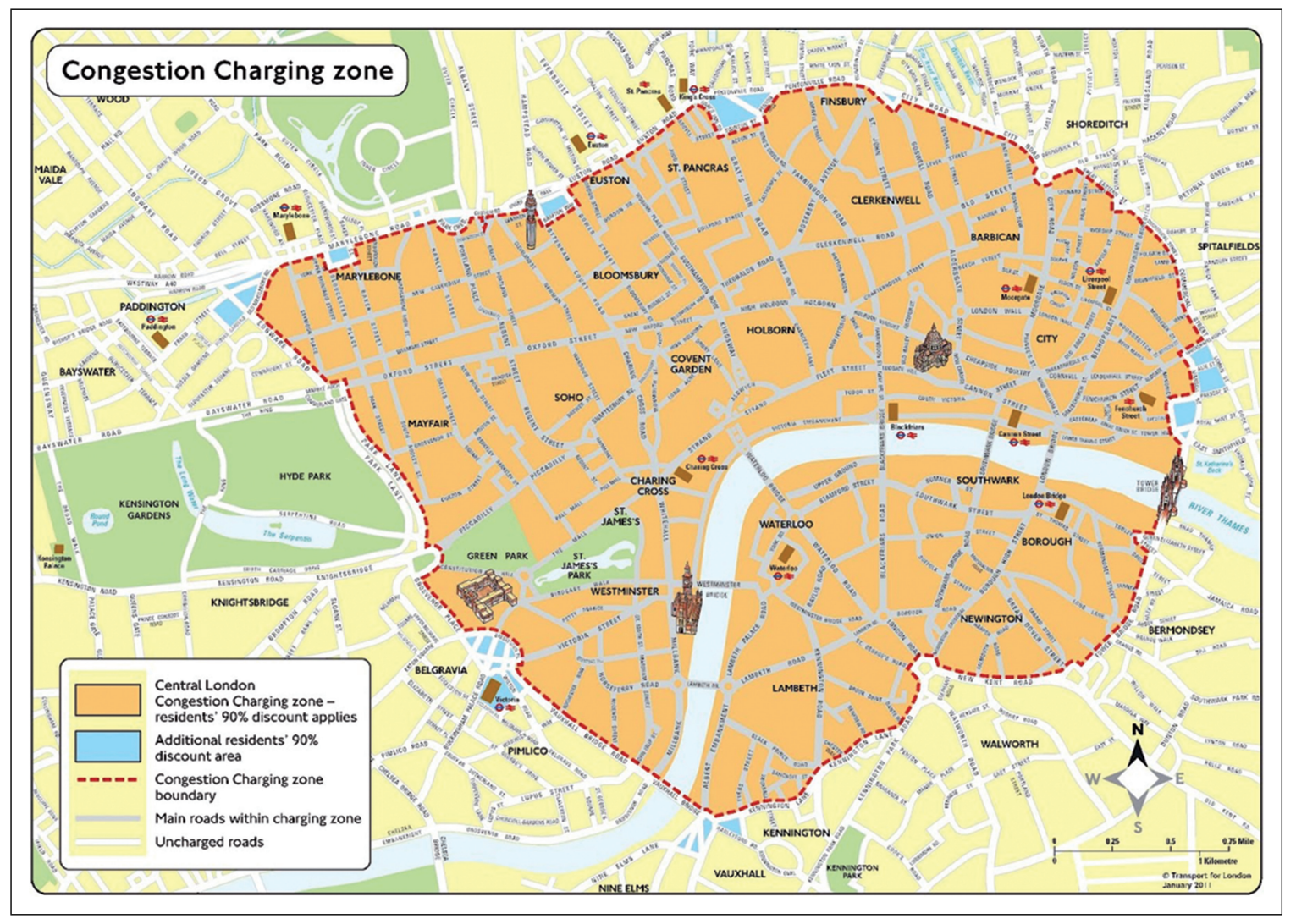

Figure 6 La zone du système LCC [17] 
(en ligne, pas SMS, par téléphone, à des magasins, ou par la poste) et il est possible de payer pour un jour, une semaine, un mois ou un an (les deux derniers à un tarif préférentiel), ou pour des dates sélectionnées dans le futur. En cas de non-paiement, une amende est émise, dont le montant varie de $£ 60$ à $£ 180$ selon le retard.

Des remises et des exemptions sont accordées pour certaines catégories de véhicules. Les motos, les taxis, les véhicules des services d'urgence, les véhicules utilisés par les personnes handicapées, et les véhicules de transport en commun sont automatiquement exonérés ; d'autre part, les véhicules «verts " (à combustible alternatif, à propulsion électrique, etc.), les minibus à plus de neuf places, les triporteurs et les véhicules de dépannage bénéficient d'une remise de $100 \%$, sujette à un enregistrement. Les habitants de la zone, ainsi que les habitants qui résident dans certains quartiers contigus aux limites de la zone, bénéficient d'une remise de $90 \%$, et un tarif réduit de £9 s'applique aux véhicules individuels et aux flottes de véhicules enregistrés dans le Système de paiement automatique (Congestion Charging Auto Pay) [1].

En ce qui concerne la signalisation, des panneaux et des marquages sur la voie indiquent quand les automobilistes approchent, entrent et quittent la zone (Figure 7) ; ceux-ci sont de plus accompagnés par des panneaux informatifs supplémentaires. Plus particulièrement, des panneaux indiquant les heures de fonctionnement sont situés près de tous les points d'entrée de la zone (a), et des panneaux démarquant la fin de la zone sont localisés à tous les points de sortie (b). À l'intérieur de la zone, des marquages rouges $« \mathrm{C} »$ et des panneaux rappelant aux conducteurs leur obligation de payer les frais (c) sont souvent placés près des points d'entrée. Des panneaux et marquages directionnels à l'approche des carrefours indiquent quelles routes mènent à la zone, et les voies menant à ces routes sont marquées par un symbole « $\mathrm{C} »$ blanc (d). Enfin, des informations sont disponibles à l'avance sur les routes d'approche principales par des panneaux situés à diverses distances de la zone et rappelant aux automobilistes les heures d'opération et le montant qu'ils doivent payer (e).

\section{Méthodologie et aspects technologiques}

La partie la plus innovante du LCC est la méthode de prélèvement de la taxe, c'est-à-dire la vérification que les frais pour tous les véhicules ayant pénétré dans la zone ont été payés. Par opposition aux systèmes de péage conventionnels, le LCC ne comprend pas de postes de péage ou de barrière, ni de ticket ou de vignette [17]. Cependant, le système est basé sur la vidéosurveillance, et repose sur la lecture précise des numéros d'immatriculation afin d'identifier et de percevoir la taxe [18].
Concrètement, il s'agit d'un réseau de 197 sites de caméras qui surveillent les points d'entrée et de sortie de la zone. Ce dispositif est complété à l'intérieur de cette dernière par des patrouilles de surveillance équipées de caméras montées sur des camionnettes. Chaque site dispose d'au moins une caméra couleur et d'une caméra monochromatique pour chaque voie de circulation surveillée : la première prend une image $d u$ « contexte » $d u$ véhicule concerné, et la seconde produit une image en gros plan de la plaque d'immatriculation. Les images saisies par les deux caméras sont des preuves d'éventuelles infractions, c'est-à-dire une compilation d'images prouvant la présence du véhicule dans la zone pendant les heures d'opération (Figure 8).

Les images saisies sont ensuite traitées par un système intégré de lecture automatisée des plaques d'immatriculation (Lapi) ; la compilation des preuves est produite in situ et est ensuite encryptée et transmise au centre de données du LCC par un canal de communication dédié à haut débit. Au centre de données, un logiciel spécialisé vérifie si le numéro d'immatriculation d'un véhicule détecté est compris dans la base de données des frais payés pour la journée concernée. Si les frais sont payés, ou si le véhicule est exempté ou est sujet à une remise de $100 \%$, l'image est effacée du système central. Une vérification finale est réalisée à minuit le lendemain en marquant les numéros d'immatriculation pour lesquels un paiement n'a pas été effectué. Les images sont ensuite vérifiées manuellement et des amendes sont émises pour les véhicules en infraction. Les dossiers de preuve des véhicules ayant reçu une amende sont conservés pendant 13 mois afin de gérer les contraventions, et sont par la suite effacés.

\section{Forces et faiblesses}

Depuis l'introduction du LCC, plusieurs études d'évaluation ont été menées, d'abord par TfL même [19], mais aussi indépendamment [20]-[23]. La majorité d'entre elles se sont concentrées presqu'entièrement sur l'étude des impacts et sur l'évaluation financière du système. Les aspects technologiques n'ont été abordés que dans peu d'études (par exemple [18]) et dans un programme d'essais techniques de TfL [24]. L'objectif de ce dernier était d'examiner comment les nouvelles technologies pourraient soutenir le système existant en réduisant ses coûts, en améliorant sa précision, et en le rendant plus convivial, mais aussi comment des systèmes plus avancés pourraient à l'avenir compléter ou même remplacer le LCC original.

Dans son rapport final en 2007 [25], le programme a conclu que, malgré les possibilités d'amélioration du système, une technologie candidate non encore entièrement développée présente un grand potentiel qui devrait permettre 


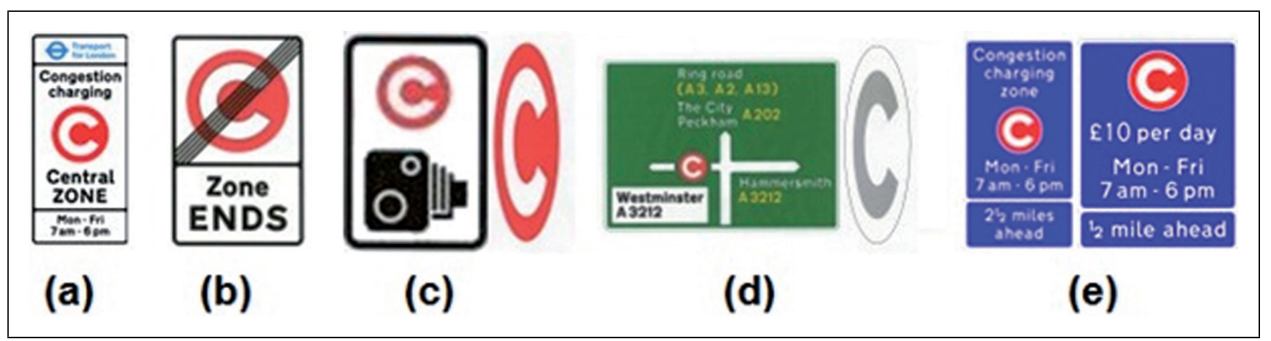

Figure 7 La signalisation du LCC [17]

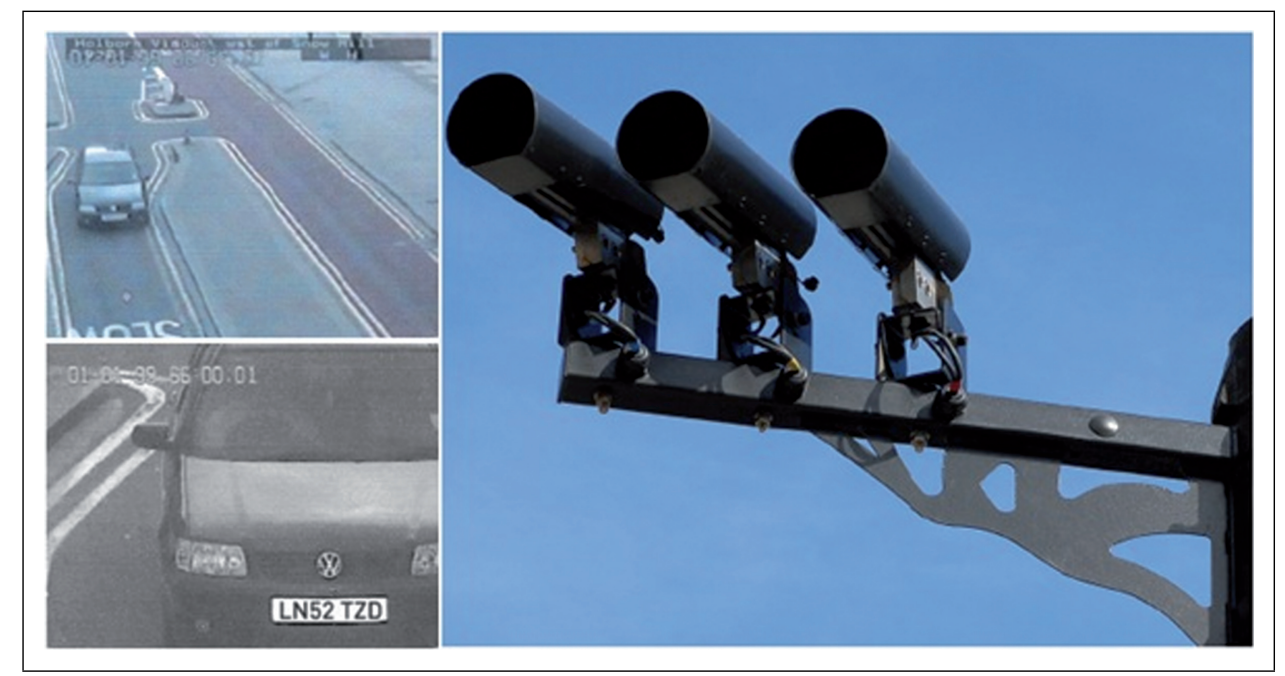

Figure 8 Les caméras du LCC et les preuves en images [17]

d'anticiper l'évolution du système. De là, et en examinant le système neuf ans après son introduction, la présente étude en évalue les forces et faiblesses et identifie les possibilités offertes par les STI coopératifs existants et émergents.

Une des forces les plus importantes du LCC est qu'il a permis de prouver la possibilité effective d'introduire un péage urbain dans une grande ville occidentale. Le péage urbain n'avait au préalable été réalisé qu'à petite échelle. Le fonctionnement du système et sa compréhension par le public sont relativement faciles; il produit des recettes régulières et fiables qui dépassent ses coûts opérationnels, et il a eu de l'effet sur le trafic, même s'il a progressivement diminué depuis son introduction. De plus, la promotion du système a été bien faite : il n'a pas provoqué des grandes protestations de la part du public, il fonctionne sans problème depuis quelques années et il est maintenant accepté comme faisant part de la vie à Londres.

Une grande réussite technologique du LCC est qu'il a démontré la possibilité d'opérer un système de péage urbain fondé uniquement sur Lapi, mais aussi la faisabilité de l'introduction d'un système avancé qui permet aux utilisateurs de payer les frais par téléphone ou sur internet. En plus, le système bénéficie d'un mécanisme de taxe assez efficace, puisque des phénomènes d' " évasion de masse »n'ont pas été observés. Le système de base de données novateur qui permet de mettre en parallèle les archives vidéos avec les identités des utilisateurs, ainsi que la conservation des données qui permet de suivre les traces des contrevenants au cas où une action est nécessaire (par exemple infliger une amende) joue un rôle important dans cette réussite. Le fait que l'utilisation de cette technologie ait permis l'introduction du système sans qu'il soit nécessaire d'installer quoi que ce soit dans le véhicule est en soi un accomplissement.

La faiblesse principale du système est son coût d'opération plus élevé que prévu. Avec une proportion entre les coûts et les recettes de $35 \%$, le LCC n'a pas été le « grand pourvoyeur » qu'on avait espéré avant son entrée en service. Ces coûts élevés résultent de la possibilité que des usagers omettent de payer la taxe à cause de la simplicité du système (un utilisateur peut pénétrer dans la zone sans en être conscient) et, par conséquent, d'importants mécanismes doivent être déployés pour les repérer. Ceci implique donc une tâche considérable supplémentaire de vérification manuelle des dossiers nonidentifiés par le système Lapi, c'est-à-dire les véhicules pour 
lesquels la lecture automatique du numéro minéralogique a échoué.

Une autre faiblesse du LCC est que le système couvre une zone relativement petite et, comme c'est un système d'impôt, la technologie associée ne peut pas évoluer simplement. Ainsi, toute expansion éventuelle du système nécessiterait l'installation d'équipements supplémentaires (caméras...) pour prélever la taxe et il faudrait en envisager l'évolution lorsqu'il sera plus rentable de passer à une nouvelle technologie plutôt que de continuer à l'utiliser en l'état. Malgré l'évolution, et bien que des mécanismes pour repérer les fraudeurs soient bien élaborés, il est toujours possible de contourner le système au cas où un véhicule n'est pas enregistré par les caméras.

En outre, une autre faiblesse du LCC est que la politique actuelle d'une taxe forfaitaire est en partie injuste pour certains automobilistes, même si ceci est un problème récurrent pour tous les systèmes de péage en cordon. Enfin, les systèmes de péage ne disposent pas actuellement d'une norme transfrontalière commune, ce qui rend leur compréhension peu évidente pour les utilisateurs. Ainsi, un automobiliste qui part d'une autre ville ou d'un pays voisin, ne dispose pas de toutes les informations sur les systèmes de taxe qu'il peut croiser pendant son voyage, à moins qu'il ne se soit lui-même documenté sur la question.

\section{Possibilités offertes par les STI coopératifs actuels}

Les STI coopératifs actuels ont un grand potentiel pour faire progresser le LCC, et les systèmes de péage urbain en général, principalement grâce aux moyens de communications entre Véhicule et infrastructure (v2i). On peut notamment citer la technologie de ce type la plus répandue : la Communication dédiée à courte portée (DSRC - Dedicated Short Range Communications) à 5,9 GHz qui utilise une infrastructure composée de balises et qui a été appliquée avec succès à plusieurs endroits au monde, comme à Singapour et à Stockholm. Un avantage de cette technologie est qu'elle ne nécessite pas de système Lapi, ce qui simplifie le mode de prélèvement de la taxe et réduit aussi le coût d'entretien des caméras. Toutefois, la Lapi peut rester en place comme système secondaire pour tracer les véhicules qui ne sont pas équipés d'une balise. Néanmoins, la technologie DSRC engendre des coûts d'installation d'infrastructure supplémentaire au bord de la route ainsi qu'un système de primes pour inciter les automobilistes à s'équiper de balises (en rappelant qu'un péage est une mesure impopulaire qui introduit des coûts additionnels).

Une autre technologie de STI coopératifs - qui peut être introduite dans le LCC et dans d'autres systèmes de péage urbain - est le système de localisation géographique dans lequel le véhicule est simplement équipé d'un appareil de localisation qui peut être tracé en fonction du temps. Cela pourrait rendre possible le prélèvement d'une taxe basée sur la durée, la distance parcourue et la position effective du véhicule. Ainsi, les frais à payer pour chaque utilisateur seraient calculés en fonction des rues traversées dans le réseau et de l'heure de passage. Une telle forme de péage serait plus attractive, car elle permettrait de fixer une taxe plus élevée pour les rues embouteillées, mais aussi de tenir compte de certains critères environnementaux (par exemple, appliquer des frais plus élevés aux endroits où les émissions de gaz doivent être absolument réduites). On pourrait ainsi envisager des systèmes de péage sophistiqués qui garantiraient de très bonnes précision et fiabilité de positionnement. C'est fondamental pour le déploiement de telles solutions car, en zone urbaine, la localisation par satellite n'est pas toujours assez précise et, dans le cas d'une zone de péage, une erreur de quelques mètres peut injustement générer une taxe pour un utilisateur. Pourtant, ce domaine a un grand potentiel de développement dans les années à venir, grâce aux nouveaux systèmes de positionnement par satellites (Galileo, modernisation GPS...), ainsi qu'aux technologies complémentaires de localisation (GPS différentiel, pseudolites, réseau de téléphonie mobile, capteurs embarqués, etc.). Ceci devrait permettre la localisation dans des endroits sans réception de signaux satellites, comme les tunnels, les canyons urbains et les parkings.

Une autre technologie de STI coopératifs pourrait potentiellement jouer un rôle important pour le LCC et les systèmes de péage urbain en général : celle de la téléphonie mobile (grâce à la connectivité Internet offerte par ces appareils au travers des réseaux GSM et $3 \mathrm{G}$, ainsi que par les réseaux $\mathrm{WiFi}$ ). Une plateforme de péage urbain soutenant plusieurs applications (comme un Smartphone), ou une application dédiée, pourrait déterminer la taxe à payer sur la base de la position de l'utilisateur et débiter automatiquement sa carte de crédit. Des problèmes techniques sont à considérer comme la localisation précise de l'utilisateur, s'assurer que l'utilisateur conduit un véhicule, la sélection d'un téléphone mobile sur lequel débiter les frais parmi ceux de plusieurs passagers d'un même véhicule.

Des possibilités sont aussi offertes par les technologies actuelles de communication de Véhicule à véhicule $(\mathrm{v} 2 \mathrm{v})$, notamment grâce aux applications qui pourraient potentiellement contribuer à des modes de prélèvement de taxe plus avancés et qui pourraient aussi servir à transmettre des données au travers de «pelotons » de véhicules sous forme d'un réseau ad-hoc afin d'estimer le niveau d'embouteillages dans une rue. Toutefois, une telle 
option semble irréalisable dans un futur proche, car des concepts de péage urbain plus simples doivent être mis en œuvre avant que des applications plus sophistiquées soient introduites.

\section{Possibilités offertes par les STI coopératifs émergents}

Plusieurs applications sont possibles par les STI coopératifs émergents du point de vue technologique, mais leur déploiement se heurte à des difficultés organisationnelles et politiques. Une technologie émergente prometteuse pour le LCC, et les systèmes de péage urbain en général, est l'utilisation d'un réseau de communication mobile ad hoc expérimental. Ce système utilise des détecteurs montés sur des réverbères qui sont continuellement en communication avec les véhicules et, par conséquent, les tracent dans une grande zone urbaine. Le potentiel de cette technologie est qu'il offre une possibilité de développer un système de péage dans une grande zone avec une assez bonne précision. Son désavantage est lié au besoin d'investir dans une infrastructure spécifique, de façon similaire à la DSRC.

Une autre technologie intéressante est une extension de la technique d'Adaptation intelligente de vitesse (Intelligent Speed Adaptation - ISA) qui offre un avertissement et un contrôle externe aux véhicules en fonction de la rue qu'ils sont en train de parcourir. Actuellement, l'ISA est effectuée en utilisant les limitations de vitesse enregistrées dans la carte numérique du système de navigation du véhicule mais, à cause des modifications régulières dans le réseau, il est très difficile de maintenir une telle base de données à jour. Pour cette raison, il est possible que l'architecture de l'ISA change dans un futur proche et se fonde sur une base de données hors du véhicule, à partir de laquelle le véhicule télécharge des mises à jour soit lors de l'arrivée dans une rue particulière, soit au début d'un trajet. Des extensions de l'ISA basées sur une telle architecture seront possibles dans chaque rue disposant d'un certificat contenant des informations propres au site (par exemple ISA, contrôle d'accès, réduction de $\mathrm{CO} 2$, etc.). La taxe de péage urbain pourrait être un attribut de ce certificat, ainsi l'automobiliste pourrait être averti par son système de navigation ou son téléphone mobile du montant du péage à payer, et effectuer le paiement le cas échéant.

\section{Perspective}

Dans les années à venir, la technologie de péage urbain deviendra plus robuste, plus facilement disponible et moins chère, et de nombreuses améliorations seront ainsi possibles. Néanmoins, et bien que la technologie soit un facteur déterminant pour le futur du LCC et d'autres systèmes, il ne faut pas sous-estimer les questions politiques, aux niveaux local et international. Les systèmes actuels étant ciblés sur les centres ville, les défis de la gestion du trafic dans les banlieues florissantes restent à aborder, surtout lorsque la répartition modale du trafic dans ces zones est en faveur de la voiture et non pas des transports en commun. De plus, un autre défi à relever est celui de l'évolution des carburants avec l'introduction de technologies à faibles émissions de $\mathrm{CO} 2$ qui pourraient rendre le déplacement en voiture plus attractif. D'un côté, on soulage le problème des émissions, mais d'un autre côté on risque d'aggraver celui de la circulation. Par conséquent, un plan plus général pour gérer globalement la circulation routière en milieu urbain s'avère nécessaire. Dans cette perspective, il faudra estimer ce qui est à traiter localement et ce qui fait partie d'une approche plus globale et qui, par conséquent, transcende les frontières.

\section{Vers un programme harmonisé de recherche}

Un des objectifs principaux de Nearctis est de définir un programme de recherche qui trouve son ancrage dans des problématiques actuelles et futures de gestion du trafic. Le choix d'une série d'études de cas représentatives, avec leurs spécifications et méthodes d'évaluation, a contribué à l'identification et à la définition des thématiques de recherche en gestion du trafic. Cette partie présente la démarche et le contenu d'un programme de recherche développé par les membres de Nearctis.

\section{Démarche}

La gestion du trafic évolue d'un problème technique isolé vers une vision plus intégrée et plus large de la gestion de la mobilité dans laquelle de multiples critères et contraintes doivent être pris en compte dans un contexte de croissance de la demande face à des problèmes accrus de congestion. En même temps, on assiste à un développement rapide des NTIC qui influent fortement sur notre société de la mobilité.

L'évolution continue des technologies et des moyens de gestion du trafic routier présente de nouvelles opportunités pour le développement et l'implémentation d'approches novatrices en gestion et suivi du trafic. Les perspectives de cette évolution ouvrent un champ de recherche innovant avec l'émergence de nouveaux objectifs et méthodologies. Afin d'anticiper l'impact de ces avancées technologiques sur le suivi et la gestion du trafic, il s'avère nécessaire d'aborder ces problématiques d'une manière systématique et dans la perspective de développer une recherche harmonisée.

Le réseau d'excellence Nearctis a donc entrepris une démarche prospective afin d'étudier le potentiel et les 
bénéfices des technologies coopératives de l'information et de la communication dans la perspective d'une amélioration des systèmes de transports routiers individuels et collectifs, de la gestion du trafic et des services d'information aux voyageurs. Un des objectifs principaux est le développement d'un programme de recherche harmonisé sur la gestion coopérative du trafic basé sur des analyses (larges et conjointes) des systèmes actuels de gestion du trafic, des technologies coopératives des NTIC, des moyens de localisation et de l'état des connaissances dans ce domaine.

Cette démarche a été réalisée en trois étapes, avec dans un premier temps un état de l'art des technologies et une identification des lacunes de recherche [1], puis dans un second temps l'élaboration du programme de recherches [4], et finalement une évaluation de ce programme.

\section{Cadre de référence d'un programme de recherche}

Le développement de ce programme de recherches s'inscrit dans un cadre comprenant des dimensions fondamentales de manière à expliciter les synergies et complémentarités des recherches futures. Ce cadre repose principalement sur des objectifs clés, des thématiques de recherches, des domaines d'expertise, des domaines de la gestion du trafic et des opportunités pour contribuer à l'innovation. On décrit ci-dessous les objectifs et les domaines d'expertise.

\section{Objectifs de recherche}

L'Union Européenne a identifié les défis majeurs à long terme de la gestion du trafic dans lesquels les systèmes coopératifs des NTIC pourraient s'insérer. Ceci a permis de sélectionner 5 objectifs clés pour le programme de recherche de Nearctis : la sécurité, les impacts sur l'environnement, l'efficacité des systèmes de transport routier (individuel et collectif), les expériences des utilisateurs et des voyageurs et les impacts sociaux, coûts et équité. En complément de ces objectifs, on a également considéré le futur programme de l'Union européenne Horizon 2020 avec les priorités suivantes : principaux challenges de la société, leadership industriel et excellence scientifique.

\section{Domaines d'expertise}

La conduite de recherches dans le domaine de la gestion $\mathrm{du}$ trafic et des systèmes coopératifs recourt à d'excellents niveaux de connaissances avec des compétences d'analyse, en ingénierie et en sciences sociales. L'efficacité des systèmes coopératifs dépend de la compréhension du comportement des usagers et des voyageurs face à l'accroissement d'informations et au déploiement de systèmes de transport plus flexibles. Pour cela, on identifie les domaines d'expertises spécifiques suivants :

- Modélisation de trafic avec l'intégration de systèmes coopératifs : parmi les buts et la diversité des modèles, on inclut les domaines clés comme la compréhension et le contrôle du système, l'évaluation, la planification des transports et des infrastructures. On identifie trois types de systèmes particuliers : les agents coopératifs individuels (conducteurs, véhicules), les systèmes coopératifs de régulation et les systèmes coopératifs intégrés (associant véhicules et systèmes de régulation).

- Optimisation et régulation : une gestion optimale du trafic et un usage adéquat de l'infrastructure doivent permettre d'assurer l'efficacité et la sécurité du transport de personnes et de biens avec un impact minimum sur l'environnement. Le déploiement rapide des NTIC aidera à l'optimisation de la gestion, dont l'efficacité dépendra des méthodes appropriées de régulation.

- Localisation et communication : le déploiement des futurs services de gestion du trafic est basé sur des nouvelles technologies de communication et de positionnement. Les applications sécuritaires demanderont des systèmes de mesures et de monitoring fiables et de haute qualité.

- Évaluation des impacts, déploiement et implémentation : les quatre facteurs importants pour le succès du déploiement des applications ITS sont : l'évaluation de l'impact, l'architecture système, l'interopérabilité et les effets de transition. En recourant à des modèles détaillés de simulation, l'évaluation de différentes stratégies considérant de nombreux critères (temps de parcours, consommation, émissions) devient possible. Ainsi, l'évolution des architectures systèmes conduit à l'amélioration de l'efficacité et de la fiabilité des différents systèmes coopératifs.

- Évaluation de la performance du système de transport routier : c'est l'un des buts à long terme du déploiement des systèmes coopératifs pour la gestion du trafic. Pour cela, on identifie trois domaines clés : l'expression et l'évaluation d'objectifs de la politique des transports, l'identification des techniques appropriées de gestion du trafic vis-à-vis de ces objectifs et le développement d'outils pour concevoir et évaluer ces mesures.

\section{Le programme de recherche harmonisé}

Le programme de recherche harmonisé de Nearctis est composé de sept thématiques dans lesquelles sont rassemblées les idées et visions de recherche développées par l'ensemble 
des partenaires [4]. Pour chaque thème, on considère la motivation, la justification, les besoins et finalement le bénéfices escomptés. Ce programme a identifié des thèmes de recherche offrant un grand potentiel de bénéfices pour les systèmes coopératifs en gestion du trafic - en laissant ouvert la possibilité d'étendre les domaines avec des sujets novateurs de recherche. On rappelle que le futur « institut virtuel » assurera la promotion de ce programme auprès des instances concernées et le concrétisera par des actions de recherche en réseau.

\section{Disponibilité accrue des communications mobiles}

La facilité d'usage et la disponibilité des communications mobiles offrent des perspectives pour diffuser des données sur l'état actuel et à court terme du trafic, les conditions météo et l'état de la route. Dans le contexte de la circulation routière, cette diffusion d'information peut se faire selon le sens du trafic, de manière à renseigner les usagers en amont afin qu'ils puissent, si nécessaire, prendre une décision adéquate.

Les terminaux mobiles sont aussi des moyens de saisie d'informations utiles à l'estimation de variables - comme la vitesse, le flux ou la densité de trafic -, d'un point de vue micro ou macroscopique. L'extraction de ces données peut servir au monitoring de réseaux de transports et aider au suivi et à la gestion du trafic.

Les communications mobiles permettent de connecter les véhicules entre eux, ainsi que les véhicules à l'infrastructure, ce qui facilite la gestion et la régulation en conditions normales et permet une détection plus rapide d'incidents. Par conséquent, l'information aux usagers est améliorée et plus efficace, offrant ainsi des moyens performants de planification ou de gestion des itinéraires.

\section{Augmentation de la disponibilité des données}

Une des conséquences de l'accroissement des communications mobiles est la capacité de fournir des données de manière extensive - ce qui permet non seulement d'estimer l'état courant du trafic, mais également de connaître les intentions des usagers. Ce type d'information devrait améliorer la gestion dynamique du trafic si les données sources sont adéquates et fiables.

L'augmentation de la disponibilité des données nécessite des recherches appropriées sur la gestion de la qualité de celles-ci, les algorithmes de fusion des données et les méthodes statistiques et d'analyse. Le fait de multiplier les sources d'information nécessite une précaution particulière dans l'intégration de nouveaux types de données (par ex. les destinations introduites par les usagers dans un navigateur) dans les modèles et système de gestion et de régulation du trafic.

\section{Amélioration de la modélisation à tous les niveaux}

La disponibilité accrue de données sur les conditions de trafic offre de nouvelles opportunités pour la modélisation à toutes les échelles. Dans la perspective du déploiement des systèmes coopératifs, le transfert de résultats entre modèles devient de plus en plus important. Disposer de données en temps réel permet de nouvelles modélisations à petite échelle en tenant compte de phénomènes locaux, mais également à grande échelle en mesurant l'impact régional des systèmes coopératifs pour la gestion du trafic.

Dans cette perspective, les problématiques de recherche incluent l'estimation en temps réel des conditions de trafic, l'amélioration de l'estimation des temps de parcours, la modélisation dynamique du comportement des usagers et le développement de modèles microscopiques tenant compte des conditions de sécurité.

\section{Besoins de communication entre systèmes autonomes}

Tout type de coopération demande des communications d'information appropriées qui doivent être transmises à temps avec un contenu adapté. Par rapport aux objectifs de la gestion du trafic et des mécanismes décisionnels associés, il s'avère indispensable d'identifier l'information adéquate qui doit être transmise entre des systèmes autonomes. Ceci est d'autant plus important lorsque les systèmes doivent coopérer efficacement. Une recherche est donc indispensable pour développer les méthodes et standards d'évaluation de la pertinence de l'information, ainsi que les stratégies de communication entre systèmes autonomes (envoi, réception, routage). Comme exemple d'application, on peut citer les stratégies coopératives pour la gestion de flottes de véhicules basées sur des plateformes de partage d'informations.

\section{Conséquence des interactions à différents niveaux}

Le recours à des systèmes de communication autonomes engendre des processus décisionnels complexes dont les impacts globaux sont difficiles à cerner. La compréhension des interactions et les effets des systèmes coopératifs doivent être approfondis afin de cerner les mécanismes qui régissent, de manière cohérente, les comportements désirés des usagers.

Ce domaine de recherche inclut les véhicules intelligents, la régulation du trafic à plusieurs échelles et l'étude des interactions entre systèmes actifs. 


\section{Parvenir à une gestion efficace à large échelle des systèmes de transport}

La gestion coordonnée des transports doit faire face aux défis de gestion à large échelle en intégrant de nouveaux outils efficaces. Ces derniers doivent permettre d'anticiper les effets globaux faisant suite à des décisions locales en matière de gestion du trafic ou d'incidents.

Ce domaine de recherche inclut la gestion d'incidents, le déplacement de piétons dans des environnements multimodaux, la gestion active du trafic autoroutier, la régulation du trafic en zone urbaine et les systèmes différenciés de taxes routières.

\section{Soutien à la politique de développement}

La mise en œuvre d'un concept de gestion coordonnée des transports nécessite de nouveaux standards et une adaptation appropriée des réglementations en vigueur. Afin d'harmoniser le développement de règles et de standards, il faut que les responsables et les organismes habilités disposent d'outils permettant l'estimation du potentiel et l'évaluation des bénéfices de ces futurs systèmes coopératifs.

Pour chacune des thématiques, les membres de Nearctis ont émis des idées de recherche plus détaillées en posant une série de questions de recherche et en décrivant les contributions attendues par rapport aux objectifs et aux domaines d'expertise décrits dans le sous-chapitre précédent. Cette approche analytique a permis d'identifier les complémentarités des thèmes de recherche, ce qui contribue à l'harmonisation de l'ensemble du programme, tout en apportant une valeur ajoutée grâce aux compétences d'un institut en réseau.

\section{Conclusions et perspectives}

Le déploiement rapide des NTIC dans les systèmes coopératifs est largement soutenu par l'industrie automobile qui, en même temps, développe de nombreux standards qui ont un impact sur les équipements d'infrastructures et de services. Les opérateurs routiers développent également leur concept de route intelligente avec des perspectives d'intégration de services et de moyens de communication entre les différents acteurs du domaine routier. Toutefois, cette évolution ne doit pas seulement être dictée par les apports de la technologie, mais doit tenir compte des questions et problématiques essentielles de gestion et de politique de la mobilité Ainsi, le programme de recherche proposé par Nearctis s'inscrit dans cette logique et devrait fournir des connaissances indispensables à une réalisation coordonnée de ces concepts de gestion du trafic soutenue par le déploiement des NTIC.
Cet article présente un volet du travail entrepris par le réseau d'excellence Nearctis en démontrant l'importance d'une recherche fondamentale sur les problématiques de modélisation et de gestion du trafic. L'établissement d'un programme de recherches harmonisé, en ayant préalablement identifié les lacunes actuelles de recherche et les cas d'applications pertinents, est un atout pour un réseau académique qui peut intéresser tous types de partenaires à participer à ces défis de recherche et de notre société. Le programme de recherches a été présenté et discuté avec les principaux partenaires lors d'ateliers, et a fait l'objet d'une analyse et d'une évaluation qui sont publiées dans le livrable D18 [26]. Les thématiques proposées s'insèrent parfaitement dans le programme proposé par l'Union Européenne à l'horizon 2020.

C'est le futur « institut virtuel» qui aura la responsabilité de promouvoir ce programme au travers de diverses actions qui devront permettre d'insérer ces thématiques dans les futurs programmes de recherche européens et nationaux. L'institut en réseau, avec ses domaines d'expertise, jouera également un rôle fédérateur en permettant la création adéquate de groupements d'instituts de recherche mixtes (public, privé) lors de la réponse à des appels à proposition. Depuis plus de quatre années, la collaboration inter-instituts fonctionne très bien et permet une excellente synergie entre scientifiques de compétences pointues et complémentaires. Le programme de mobilité des chercheurs et les ateliers Nearctis y ont grandement contribué. Ceci s'est déjà vérifié lors de montages de projets (Action Cost, Marie Curie ITN) et se poursuivra avec la nouvelle structure. Parmi les actions de dissémination, on peut relever la volonté des membres du réseau de participer à un débat public large en étant représenté dans des groupes de travail européens comme le working group on automation et smart cities. Pour ce faire, les représentants de Nearctis s'appuient sur les livrables qui ont été produits concernant les futurs sujets de recherche possibles (D14, D18). Nearctis commence à être un interlocuteur reconnu par la Commission puisque la DG RTD lui a demandé des éléments pour rédiger la première série d'appels à projets pour Horizon 2020.

Finalement, l'institut en réseau proposera un programme de formation de haut niveau, ainsi que des outils éducatifs, en gestion du trafic et systèmes coopératifs pour les chercheurs et praticiens, dans la continuité du succès des écoles d'été de Nearctis.

En conclusion, la perspective de développer un institut de référence en réseau sur ce thème important pour notre société de mobilité est très prometteuse car cette plateforme scientifique mettra à disposition de nombreuses compétences, ressources et connaissances au profit d'une large communauté de responsables des questions de mobilité et de gestion du trafic routier. 


\section{Références}

1. Nearctis (2010) deliverable D6, Preliminary report on the review of the state of knowledge and capability in relevant technologies.

2. Nearctis (2009) deliverable D7, Review of available case studies and related scientific knowledge.

3. Nearctis (2010) deliverable D8, Identification of successes, gaps and potential for improvements in traffic management applications.

4. Nearctis (2011) deliverable D14, The Harmonised research programme.

5. Nearctis (2012) deliverable D15, Specification and evaluation approaches for possible case studies.

6. Ministère néerlandais de l'infrastructure et de l'environnement (2008) Beleidskader Benutten. Eén van de pijlers voor een betere bereikbaarheid. Achtergronddocument (en néerlandais). Ministère néerlandais de l'infrastructure et de l'environnement, Direction général du transport des voyageurs.

7. van Kooten J, Martens G (2008) Praktijkproef Amsterdam, GGB gebiedsgerichte uitwerking (en néerlandais). Arane consultants en trafic et transport.

8. Beijer P, Hoogendoorn-Lanser S, Hoogendoorn SP, Buijze H, Westerman M, Meurs H, Polderdijk S, van Kooten J, Schaap V, van Konijnenburg P (2009) Praktijkproef Verkeersmanagement Amsterdam, Proof of Concept Eindrapport (en néerlandais), Ministère de l'infrastructure et de l'environnement, Pays-Bas.

9. Daganzo CFN, Geroliminis N (2008) An analytical approximation for the macroscopic fundamental diagram of urban traffic. Transportation Research Part B 42: 771-781.

10. Geroliminis N, Daganzo CF (2008) Existence of urban-scale macroscopic fundamental diagrams: Some experimental findings, Transportation Research Part B 42: 759-770.

11. Province Noord Holland, Ministère des travaux publics, région d'Amsterdam et la municipalité d'Amsterdam (2005) Netwerkvisie Noord Holland (en néerlandais).

12. Papageorgiou M, Haj-Salem H, Blosseville JM (1991) ALINEA: A Local Feedback Control Law for on-ramp-metering. Transportation Research Record 1320: 58-64.
13. Papageorgiou M, Haj-Salem H, Middelham F (1997) ALINEA local ramp-metering: Summary of field results. Transportation Research Record 1603: 90-98.

14. Papageorgiou M, Ben-Akiva M, Bottom J, Bovy PHL, Hoogendoorn SP, Hounsell NB, Kotsialos A, McDonald M (2007) Chapter 11 ITS and Traffic Management. Handbooks in Operations Research and Management Science 14: 715-774.

15. Hoogendoorn SP, Daamen W, Yuan Y (2009) Ex-ante evaluatie gecoördineerde toeritdosering Amsterdam: Tuning, evaluatie en aanpassing van het HERO algoritme (en néerlandais). Delft, Netherlands.

16. Yuan Y (2008) Coordination of Ramp-metering Control in Motorway Networks, Master thesis, ITS Edulab, Delft, Pays-Bas.

17. Transport for London, Site officiel, http://www.tfl.gov.uk, 2012.

18. Blythe PT (2005) Congestion charging: Technical options for the delivery of future UK policy. Transportation Research A 39: 571-587.

19. Transport for London (2008) Congestion Charging 6th Annual Impacts Monitoring Report.

20. Prud'homme R, Bocarejo JP (2005) The London congestion charge: a tentative economic appraisal, Transport Policy 12: 279-287.

21. Mackie P (2005) The London congestion charge: A tentative economic appraisal. A comment on the paper by Prud'homme and Bocajero, Transport Policy 12: 288-290.

22. Raux C (2005) Comments on 'The London congestion charge: a tentative economic appraisal, Transport Policy 12: 368-371.

23. Schmöcker JD, Fonzone A, Quddus M, Bell MGH (2006) Changes in the frequency of shopping trips in response to a congestion charge, Transport Policy 13: 217-228.

24. Transport for London (2005) London Congestion Charging Technology Trials Stage 1 Report.

25. Transport for London (2005) Congestion Charging Technology Trials Stage 3 Final Report.

26. Nearctis (2012) deliverable D18, Evaluation and assessment of likely benefits of the harmonised research programme. 